\title{
Influence of the Tumor Microenvironment on Cancer Cells Metabolic Reprogramming
}

\author{
Victoire Gouirand ${ }^{1,2,3,4}$, Fabienne Guillaumond ${ }^{1,2,3,4}$ and Sophie Vasseur ${ }^{1,2,3,4 *}$ \\ ${ }^{1}$ Centre de Recherche en Cancérologie de Marseille (CRCM), UMR 1068, Institut National de la Santé et de la Recherche \\ Médicale, Marseille, France, ${ }^{2}$ Institut Paoli-Calmettes (IPC), Marseille, France, ${ }^{3}$ Unité Mixte de Recherche (UMR 7258), \\ Centre National de la Recherche Scientifique (CNRS), Marseille, France, ${ }^{4}$ Université Aix-Marseille U105, Marseille, France
}

\section{OPEN ACCESS}

Edited by:

Sarah-Maria Fendt, VIB KU Leuven Center for Cancer Biology, Belgium

Reviewed by:

Daniela Gaglio,

Istituto di Bioimmagini e Fisiologia Molecolare (CNR),

Bin-Zhi Qian,

University of Edinburgh, United Kingdom

*Correspondence: Sophie Vasseur sophie.vasseur@inserm.fr

Specialty section: This article was submitted to Molecular and Cellular Oncology,

a section of the journal

Frontiers in Oncology

Received: 12 February 2018 Accepted: 03 April 2018 Published: 19 April 2018

Citation:

Gouirand V, Guillaumond F and Vasseur S (2018) Influence of the Tumor Microenvironment on Cancer Cells Metabolic Reprogramming.

Front. Oncol. 8:117. doi: 10.3389/fonc.2018.00117
As with castles, tumor cells are fortified by surrounding non-malignant cells, such as cancer-associated fibroblasts, immune cells, but also nerve fibers and extracellular matrix. In most cancers, this fortification creates a considerable solid pressure which limits oxygen and nutrient delivery to the tumor cells and causes a hypoxic and nutritional stress. Consequently, tumor cells have to adapt their metabolism to survive and proliferate in this harsh microenvironment. To satisfy their need in energy and biomass, tumor cells develop new capacities to benefit from metabolites of the microenvironment, either by their uptake through the macropinocytosis process or through metabolite transporters, or by a cross-talk with stromal cells and capture of extracellular vesicles that are released by the neighboring cells. However, the microenvironments of primary tumor and metastatic niches differ tremendously in their cellular/acellular components and available nutrients. Therefore, cancer cells must develop a metabolic flexibility conferring on them the ability to satisfy their biomass and energetic demands at both primary and metastasis sites. In this review, we propose a brief overview of how proliferating cancer cells take advantage of their surrounding microenvironment to satisfy their high metabolic demand at both primary and metastasis sites.

Keywords: microenvironment, metabolism, tumor, metastasis, protein scavenging, fibroblasts

\section{INTRODUCTION}

Cellular heterogeneity of solid tumors strongly impacts tumor progression. This abundant heterocellularity drives the nature and abundance of the components of the extracellular matrix (ECM) and, for some cancers, makes tumor cells the minor cell type in terms of cellular amount. It appears that tumor cells take advantage of this dense microenvironment and are engaged in a complex dialog with their surrounding cells. A multitude of studies have emerged to dissect the inflammatory, metabolic, or oncogenic nature of the dialog between different cell types in tumors and have improved our knowledge of the various communication modes between cells; physical interactions, secreted molecules, extracellular vesicles (EVs), etc. In this review, we highlight how the stroma [mainly cancer-associated fibroblasts (CAFs)]-tumor cell metabolic axis increases the metabolic performance of tumor cells in addition to the cell's autonomous metabolic pathways. We also discuss how tumor cells recycle some metabolites, considered until now as "metabolic wastes," to support their biosynthetic and bioenergetic needs. Finally, we point out the metabolic plasticity that metastatic tumor cells acquire to adapt to the microenvironments of both the primary and metastasis sites. 


\section{METABOLIC COMMUNICATION BETWEEN TUMOR CELLS AND THEIR NEIGHBORING CELLS}

Cancer-associated metabolic remodeling is not restricted to malignant cells but is also found in tumor-surrounding, nontransformed stromal cells. This stromal metabolic reprogramming is dictated by the tumor cells and, as a feedback loop, microenvironmental cells drive metabolic changes in tumor cells and/or provide metabolic resources required for tumor growth. CAFs are the most prominent cell type in the tumor microenvironment (TME) $(1,2)$ and have emerged as key components of the stromal-epithelial metabolic coupling. In pancreatic cancer cells, Sherman et al. have shown through a transcriptomic approach that soluble cues from patient-derived CAFs induce deep metabolic alterations that are similar to those driven by K-RAS. The metabolic pathways found the most enriched in stroma-activated tumor cells are associated with steroid and unsaturated fatty acid biosynthesis, and also with glycolysis and gluconeogenesis.
Moreover, stromal cues increase flux through glycolysis and pentose phosphate pathway (PPP) and enhance tricarboxylic acid (TCA) cycle intermediates. Hence, the stroma induces genomic and metabolic responses that strengthen pancreatic tumor progression (3). A recent in situ study analyzing single cell enzymatic activities in intact tumor tissues showed that CAFs have a higher glycolytic activity than the different subtypes of breast tumor cells (4). CAFs metabolize glucose through anaerobic glycolysis and export lactate which is then taken up by oxidative cancer cells to increase their tumorigenic potential (5), a phenomenon coined the "reverse Warburg effect" which is also reported in other cancers (6) (Figure 1). The CAFs's-enhanced glycolysis is induced by cancer cells in response to hypoxic or oxidative stress injuries $(7,8)$ and is not a general adaptive metabolic feature of CAFs. Indeed, even if pancreatic-CAFs are prone to glycolysis (9), the lactate is secreted at a lower rate than alanine, a non-essential amino acid (NEAA) (10). Secretion of alanine by CAFs results from autophagy-induced protein breakdown in these cells and, once taken up by cancer cells, it has an unusual metabolic rate. Instead of contributing to protein synthesis, alanine is converted

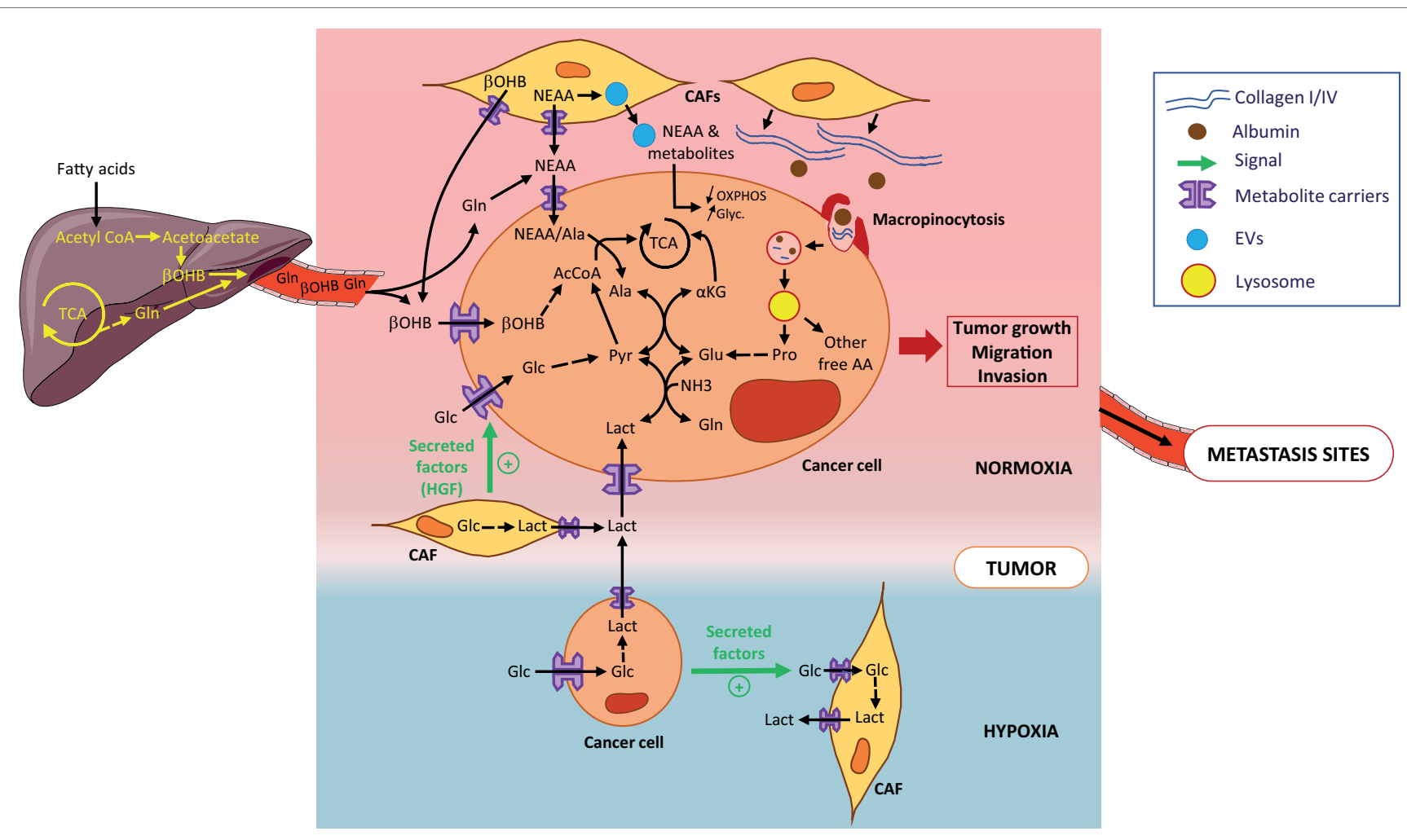

FIGURE 1 | Metabolic symbiosis and recycling in primary tumors. Tumor cells obtain metabolites and AA either from the circulation or from the local microenvironment. Metabolites, such as $\beta-\mathrm{OHB}$ or Gln are released into the circulation by the liver and reach the normoxic tumor (pink zone). At the primary tumor site, AA and other metabolites are locally released by cancer-associated fibroblats (CAFs) (yellow cells). Tumor cells (light brown cells) directly take up AA and metabolites through metabolite carriers or indirectly by (1) uptake of metabolites-loaded EVs from CAFs and (2) macropinocytosis of extracellular matrix such as collagen or scavenging of macromolecules such as albumin. Macropinosomes are internalized and fused with lysosomes where collagen and albumin are degraded into free proline and free AA, all released in the cytosol. Lactate secreted by hypoxic tumor cells (localized in blue zone) is taken up by normoxic tumor cells through the metabolic symbiosis. The most part of AA and metabolites provided to tumor cells are used to contribute to the TCA. All are shown to contribute to tumor growth and metastatic potential of tumor cells. Abbreviations: AAs, amino acids; NEAAs, non-essential amino acids; Ala, alanine; Glc, glucose; Pyr, pyruvate; Lact, lactate; $\alpha \mathrm{KG}, \alpha$-ketoglutarate; Glu, glutamate; Gln, glutamine; $\beta$-OHB, $\beta$-hydroxybutyrate; EVs, extracellular vesicles; TCA, tricarboxylic cycle; OXPHOS, oxidative phosphorylation; Glyc, glycolysis. 
into pyruvate in the mitochondria to provide energy and lipids essential to pancreatic-ductal adenocarcinoma (PDAC)-cell survival and growth. In breast tumors, CAFs release kynurenine, a tumor-promoting metabolite arising from tryptophan breakdown, whose synthesis is increased in response to tumor-derived lipid mediators (prostaglandin E2) (11). In these tumors, transcriptional profiles of caveolin-1-deficient fibroblasts reveal an over-expression of ketogenic genes promoting ketone bodies (KB) production. Hence, CAFs contain a pool of KB that can be used as an energy source by oxidative breast tumor cells, and that can promote breast tumor growth (Figure 1) (12). CAFs also mediate tumor cells' metabolic reprogramming in a paracrine manner through diffusible or EV molecules. CAF-derived EVs promote a metabolic switch from mitochondrial oxidative phosphorylation (OXPHOS) to aerobic glycolysis to satisfy prostate and pancreatic tumor cell needs in ATP (Figure 1). Hence, EVs increase reductive glutamine's contribution to lipogenic acetyl-CoA cycle and the use of extracellular acetate as an additional carbon source for fatty acid synthesis. These metabolic changes are induced by intra-EV metabolites and miRNA-targeting OXPHOS genes (13). CAF-secreted cytokines, such as hepatocyte growth factor, have also been reported to favor glucose uptake in human breast tumor cells by increasing glucose transporter 1 levels (14) (Figure 1).

In tumors, nutrient-deprived conditions strengthen the stromal-epithelial metabolic shares. Under glutamine restriction, patient-derived CAFs survive and undergo a metabolic reprogramming leading to an increase in glutamine synthesis. Enhanced glucose entry into the TCA cycle, along with amino acid (AA) and lactate intake, contribute to the carbon supply for glutamine synthesis, while the nitrogen donors are branchedchain amino acids, NEAA, and ammonia. Once secreted and taken up by ovarian cancer cells, glutamine rescues tumor growth by increasing expression of genes involved in cell cycle, fatty acid, and nucleotide synthesis (15). Under AA and pyruvate starvation, CAFs enhance the packaging of cargo metabolites into EVs to rescue prostate and pancreatic tumor cell survival (13). As metabolites present in CAF-EVs are in their active form, their impact on pancreatic cell proliferation is instantaneous and strong (e.g., exosomal metabolites contribute to one-third of the TCA cycle flux), but short term (16). Interestingly, the EV-metabolite supply is K-RAS-independent (13) suggesting that CAF-mediated metabolic remodeling may be a common trait of cancers.

In addition to metabolic dialog with stromal cells, tumors cells exchange diverse metabolites between one another, a phenomenon initially referred to as metabolic symbiosis. One of the most described processes of metabolic symbiosis relies on the potential of malignant cells to increase their glucose consumption and produce large amounts of lactate through aerobic glycolysis (the Warburg effect). In hypoxic tumors, such as PDAC, large amounts of lactate secreted by highly glycolytic hypoxic cells are taken up by normoxic neighboring tumor cells and promote their proliferation. Lactate thereby contributes to the metabolic symbiosis between both hypoxic and normoxic cellular compartments of the tumor (17) (Figure 1). In non-small-cell lung cancers, Faubert et al. showed that tumor cells also metabolize lactate, proving lactate's contribution as a fuel of the TCA cycle exceeds that of glucose in vivo (18). Treatment of pancreatic neuroendocrine, breast and renal cancers with angiogenic inhibitors induces regionalization of these tumors into hypoxic and normoxic zones and force normoxic cells, in close proximity to hypoxic ones, to operate a metabolic symbiotic shift toward the use of lactate diffusing from hypoxic regions. Interestingly, lactate catabolism by normoxic tumor cells is branched to the glutamine metabolism through lactate-derived pyruvate transamination. This reaction allows the production of alanine and $\alpha$-ketoglutarate, thereby fueling the TCA cycle (Figure 1). This symbiotic metabolism is dependent on mTOR signaling as treatments of tumors with a combination of angiogenic inhibitors and the mTOR inhibitor rapamycin suppresses lactate catabolism by normoxic cells. Normoxic cells switch their metabolism back to a more glycolytic phenotype to the detriment of hypoxic cells which are then devoid of bio-available glucose (19).

Interestingly, lactate not only participates in metabolic processes but also contributes to oncogenic signaling pathways. This function is not restricted to lactate as many by-products of metabolic pathways act as signaling molecules (20). This notion of the dual function of metabolites, being involved in metabolic and oncogenic signaling, strengthens the tight association between tumor metabolism, cell cycle dysregulation, and aberrant cell proliferation. Interestingly, these processes are directed by the circadian clock which governs biological rhythms for tissue homeostasis $(21,22)$. Hence, an integrated and dynamic view of how metabolic pathways and cell cycle machinery interact with the circadian clock in the context of tumor progression deserves to be better explored, and would help to develop efficient metabolic therapeutic strategies as circadian timing of drug administration impacts both the efficacy and the toxicities of most pharmacotherapies (23).

\section{METABOLIC RECYCLING}

Increased hetero-cellularity of the TME is associated with substantial ECM deposition. This densification of the ECM during tumor growth is associated with changes in stiffness, elasticity, and mechanical properties of the microenvironment but also increases the richness of macromolecules surrounding the tumor cells. Hence, the TME becomes a nutrient supply center for the tumor cells as it is composed of abundant macromolecules, such as collagen, hyaluronan, fibronectin, albumin, lipids, etc. To take advantage of this enriched microenvironment and optimize the use of the macromolecules, tumor cells use macropinocytosis, a non-selective endocytic process, to take up extracellular components and internalize them into vesicles. Products derived from successive degradation of the vesicles' contents are released in the tumor cells' cytosol as ready-to-use nutrients. Macropinocytosis occurs in several types of cancers and is largely described as a central process of acquisition of nutrients by PDAC cells. In vivo, inhibition of macropinocytosis impedes the growth of subcutaneous xenografted pancreatic tumors (24). Vander Heiden's lab also demonstrated that PDAC satisfies its avidity for macromolecules such as albumin using macropinocytosis and locally increases its breakdown to derive pools of AAs (25). In this context, K-RAS appears to be a main oncogenic driver of this process. In vitro, K-RAS mutant PDAC cells compensate for a lack in AAs 
in culture medium and maintain a high proliferative rate due to their capacity to recover the needed AAs pools from scavenged and catabolized albumin (26). Interestingly, the ability of spontaneous PDAC to take up macromolecules is not only restricted to albumin but also includes fibronectin. Recently, we also showed that PDAC cells, when deprived of nutrients, scavenge environmental collagen which appears essential for their survival especially under glucose deprivation, the condition in which macropinocytosis is activated. Subsequent digestion of collagen supplies PDAC cells with pools of proline, a main component of collagen molecules. Proline catabolism by PRODH1 enriches the cells with TCA intermediates, especially under nutrient deprivation, and appears to be a promoting metabolic pathway of PDAC growth (Figure 1) (27).

Proliferative tumor cells can also recycle metabolites found in the circulation, such as acetate and KB. Both have been described to be carbon sources to supply the tumor with energy and biomass. Breast cancer cell lines consume large amount of acetate when subjected to metabolic stress and hypoxia to synthesize fatty acids and supply the membrane with phospholipids. AcylCoA short chain synthetase 2 promotes the acetate uptake and produces the acetate-derived acetyl-CoA pool needed for fatty acids synthesis (28). KB represented by acetone, acetoacetate, and $\beta$-hydroxybutyrate $(\beta$-OHB) constitute another family of metabolites that are usable by tumor cells. In physiological conditions, $\mathrm{KB}$ are produced in liver, released into the circulation and reach tissues/organs to supply cells in biomass and energy especially in low glucose condition. $\mathrm{KB}$ catabolism (ketolysis) consists in oxidation of $\beta-\mathrm{OHB}$, the most abundant $\mathrm{KB}$ in the circulation, to form acetoacetate which is converted by 3 -oxoacid CoA-transferase 1 (OXCT1) to produce successively acetoacetylCoA and 2 molecules of acetyl-CoA. The latter directly fuels the TCA cycle and supplies cells with ATP. In human hepatocellular carcinoma (HCC), tumor cells overexpress OXCT1 when nutrient-deprived, suggesting that these cells, contrary to the normal hepatocytes counter-part which synthesize and produce $\mathrm{KB}$, catabolize $\beta$-OHB when they are nutrient stressed. Indeed, OXCT1 favors $\beta$-OHB uptake by HCC cells which use $\beta$-OHB to fuel their TCA cycle and produce ATP to promote their proliferation (Figure 1). Moreover, OXCT1 expression in HCC cells is dependent on the mTORC2-AKT-SP1 signaling axis and induction of ketolysis by OXCT1, by supplying cells with ATP, suppresses AMPK activation upon nutrient starvation, avoiding the deleterious excessive autophagy and promoting HCC cell survival and proliferation (29). In melanoma and leukemia driven by the BRAF-V600E mutation, acetoacetate levels are increased. In tumor cells, acetoacetate enhances BRAF-V600E binding to MEK1 and thereby activates the MEK-ERK signaling axis and contributes to BRAFV600E tumor growth $(30,31)$. In breast cancer, tumor cells, by increasing the number of MCT2 transporters at the plasmic membrane, are also able to take up $\beta$-OHB produced by adipocytes localized at the tumor site. This uptake favors the clonogenic potential of MCT2 positive tumor cells as well as their capacity to form a tumor mass in vivo (32).

Ammonia recycling by tumor cells is another example of how cancer cells benefit from the capture of metabolites released either into the TME by neighboring cells or in to the circulation by the liver. Ammonia produced during the conversion of glutamine to glutamate was, until recently, considered as waste. In a metabolic symbiosis context, Spinelli et al. recently demonstrated that the recycling of ammonia maximizes nitrogen utilization by glutamate dehydrogenase for glutamate synthesis from $\alpha$-ketoglutarate. Glutamate then contributes to proline or aspartate synthesis. Importantly, the authors showed that this recycling accelerates proliferation of breast tumors (33). Hence, ammonia and $\mathrm{KB}$ recycling by tumors highlights the need to consider the interactions, in the pathophysiologic context of cancer, between tumors and the rest of the host organism. It is then crucial to integrate the metabolites exchange between the tumor and metabolic organs/tissues of the host in ex vivo studies of the metabolic reprogramming of tumors. Although the set up of in vitro and in vivo experimental models to consider such metabolic communication appears ambitious and complex, it emerges nowadays as a necessity to take tumor metabolism studies to a new level and improve the relevance of translational studies for effective metabolic therapies (34). In line with this, mathematic models of the tumor-organs metabolic interactions become essential tools in establishing experimental models.

\section{METABOLIC FLEXIBILITY OF METASTATIC CELLS}

The metastasis sites' microenvironment retains some similarity to the primary site for components such as collagens, hyaluronan, and smooth muscle actin-expressing cells (35), but also differs from the primary microenvironment in the level of their cellular/ acellular components and available nutrients. Hence, tumor cells must develop a metabolic plasticity to satisfy their biomass and energetic demands required for their proliferation in both primary and metastasis sites. It is, therefore, important to highlight a few studies revealing the metabolic programs of metastatic cells according to their tissue of origin and/or to the metastasis site microenvironment. Christen and collaborators revealed that lung interstitial fluid has increased levels of pyruvate compared to blood plasma resulting in higher pyruvate levels in lung metastases compared to primary breast tumors. Consequently metastatic breast cancer cells take advantage of the pyruvate availability in their metastatic environment by increasing pyruvate carboxylasedependent anapleurosis compared to primary tumor cells (36) (Figure 2). As a metabolic organ, the liver produces a plethora of metabolites usable by colonizing metastatic tumor cells, creatine being one of them. Creatine is phosphorylated by creatine kinase (CK), resulting in phosphocreatine, useful to shuttle high energetic phosphate when it enters into cells to produce ATP from ADP when energetic needs exceed ATP synthesis. When colon cancer cells metastasize in the liver, they must face the new environmental hypoxic conditions. Secretion of the brain-type $\mathrm{CK}(\mathrm{CKB})$ by colon metastatic cells into the extracellular space of the liver allows production of phosphocreatine from extracellular ATP and creatine. Phosphocreatine uptake by liver-disseminated metastatic cells supplies them with ATP to survive the hypoxic microenvironment (Figure 2). Consequently, inhibition of $\mathrm{CKB}$ in disseminated colon cancer cells impedes metastatic 


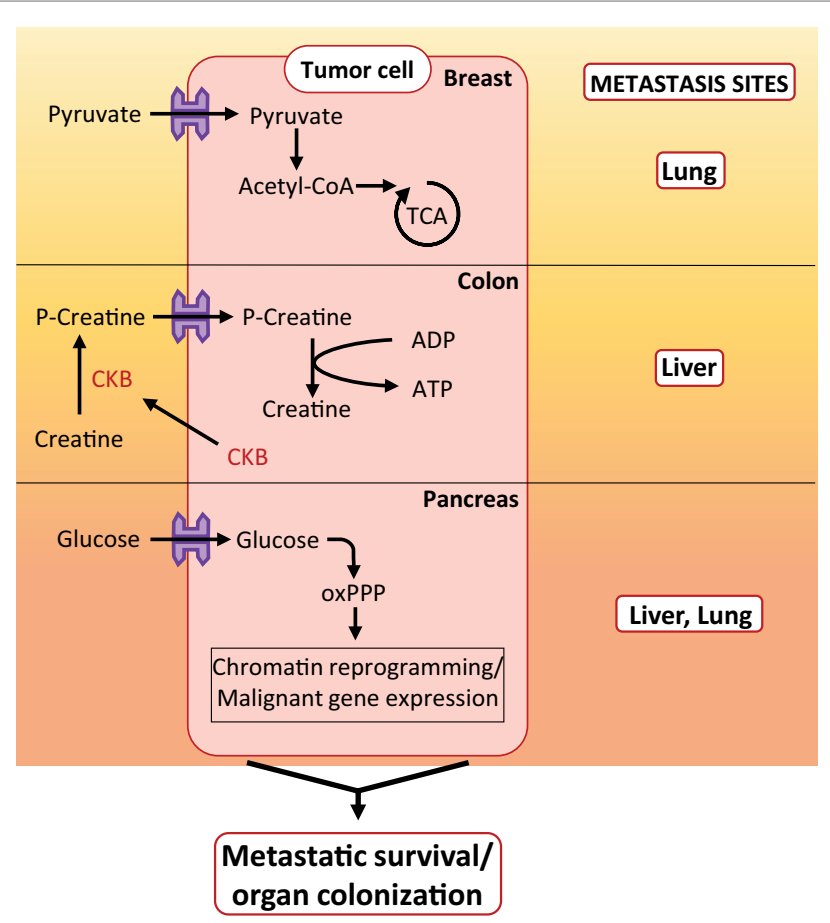

FIGURE 2 | Metabolic phenomenon occurring in metastasis sites. Example of metabolic pathways activated in breast, colon, and pancreatic metastatic cells during colonization of lung, liver, and liver/lung, respectively. Abbreviations: oxPPP, oxidative branch of the pentose phosphate pathway; $\mathrm{CKB}$, brain-type creatine kinase.

colonization of the liver. Inhibition of $\mathrm{CKB}$ and the creatine transporter in PDAC cells also limits their metastatic potential, highlighting that a common metabolic targeting of disseminated cells originating from different gastrointestinal organs is feasible to abolish metastatic progression (37). Interestingly, during the evolution of localized PDAC toward a metastatic disease, a widespread epigenetic reprogramming occurs. Moreover, metastasis at distant sites appears to be dependent on the oxidative branch of the PPP (oxPPP). Indeed, 6-phosphogluconate dehydrogenase, the enzyme responsible for nucleotide synthesis through the oxPPP, not only controls tumor growth at metastasis sites but also governs chromatin reprogramming as well as malignant gene expression. This illustrates that epigenetic events leading metastatic PDAC progression are dependent on the metabolic reprogramming of metastatic cells (38) (Figure 2).

\section{REFERENCES}

1. De Wever O, Demetter P, Mareel M, Bracke M. Stromal myofibroblasts are drivers of invasive cancer growth. Int J Cancer (2008) 123(10):2229-38. doi:10.1002/ijc.23925

2. Leca J, Martinez S, Lac S, Nigri J, Secq V, Rubis M, et al. Cancer-associated fibroblast-derived annexin A6+ extracellular vesicles support pancreatic cancer aggressiveness. J Clin Invest (2016) 126(11):4140-56. doi:10.1172/ JCI87734

3. Sherman MH, Yu RT, Tseng TW, Sousa CM, Liu S, Truitt ML, et al. Stromal cues regulate the pancreatic cancer epigenome and metabolome. Proc Natl Acad Sci U S A (2017) 114(5):1129-34. doi:10.1073/pnas.1620164114
In metastasis sites, tumor cells also influence the CAFs' metabolic activity. Indeed, breast cancer cells transfer miR-122 through EVs to resident fibroblasts in lung pre-metastatic niches. By reducing glucose consumption by fibroblasts, miR-122 increases glucose availability to tumor cells (39). Hence, metabolic remodeling of stromal cells in metastasis sites provides favorable "soil" for seeding and growth of cancer cells.

\section{CONCLUSION}

Metabolic cooperation between the TME and cancer cells contributes to tumor growth, especially in nutrient- or oxygen-deprived microenvironments. As a consequence, targeting the metabolism of stromal cells impedes tumor progression to the same extent as targeting the tumor cells' metabolic mediators. Therefore, when considering metabolic targeting of tumors as an anti-cancer therapy, targeting only the cancer cell autonomous metabolism would not be sufficient. Moreover, we highlight in this review that the nurturing microenvironment supplies tumor cells with macromolecules or metabolites and fuels metabolic pathways especially in stressful conditions. Fortunately, mechanisms used by tumor cells for the uptake of such environmental nutrients are being progressively uncovered. Development of therapeutic metabolic approaches must, therefore, take into account that the metabolic reprogramming of tumor cells is flexible and evolves along with microenvironmental changes. As such, a unique metabolic therapeutic window is not conceivable. This highlights the need to develop combined metabolic targeting to circumvent tumor metabolic plasticity and abolish tumor progression in the long term.

\section{AUTHOR CONTRIBUTIONS}

VG, FG, and SV conceived, organized, and wrote the manuscript.

\section{ACKNOWLEDGMENTS}

We thank Kendra Delicaet for editing of the manuscript.

\section{FUNDING}

This work was supported by a French National League against Cancer grant, a French Association for Cancer Research "ARC" grant, and a National Institute of Cancer "INCa" grant (grant 2015-115).

4. Miller A, Nagy C, Knapp B, Laengle J, Ponweiser E, Groeger M, et al. Exploring metabolic configurations of single cells within complex tissue microenvironments. Cell Metab (2017) 26(5):788-800.e786. doi:10.1016/j.cmet.2017.08.014

5. Romero IL, Mukherjee A, Kenny HA, Litchfield LM, Lengyel E. Molecular pathways: trafficking of metabolic resources in the tumor microenvironment. Clin Cancer Res (2015) 21(4):680-6. doi:10.1158/1078-0432.CCR-14-2198

6. Fu Y, Liu S, Yin S, Niu W, Xiong W, Tan M, et al. The reverse Warburg effect is likely to be an Achilles' heel of cancer that can be exploited for cancer therapy. Oncotarget (2017) 8(34):57813-25. doi:10.18632/oncotarget.18175

7. Danhier P, Banski P, Payen VL, Grasso D, Ippolito L, Sonveaux P, et al. Cancer metabolism in space and time: beyond the Warburg effect. Biochim Biophys Acta (2017) 1858(8):556-72. doi:10.1016/j.bbabio.2017.02.001 
8. Fiaschi T, Marini A, Giannoni E, Taddei ML, Gandellini P, De Donatis A, et al. Reciprocal metabolic reprogramming through lactate shuttle coordinately influences tumor-stroma interplay. Cancer Res (2012) 72(19):5130-40. doi:10.1158/0008-5472.CAN-12-1949

9. Shan T, Chen S, Chen X, Lin WR, Li W, Ma J, et al. Cancer-associated fibroblasts enhance pancreatic cancer cell invasion by remodeling the metabolic conversion mechanism. Oncol Rep (2017) 37(4):1971-9. doi:10.3892/or.2017. 5479

10. Sousa CM, Biancur DE, Wang X, Halbrook CJ, Sherman MH, Zhang L, et al. Pancreatic stellate cells support tumour metabolism through autophagic alanine secretion. Nature (2016) 536(7617):479-83. doi:10.1038/nature19084

11. Chen JY, Li CF, Kuo CC, Tsai KK, Hou MF, Hung WC. Cancer/stroma interplay via cyclooxygenase-2 and indoleamine 2,3-dioxygenase promotes breast cancer progression. Breast Cancer Res (2014) 16(4):410. doi:10.1186/ s13058-014-0410-1

12. Martinez-Outschoorn UE, Lisanti MP, Sotgia F. Catabolic cancer-associated fibroblasts transfer energy and biomass to anabolic cancer cells, fueling tumor growth. Semin Cancer Biol (2014) 25:47-60. doi:10.1016/j. semcancer.2014.01.005

13. Zhao H, Yang L, Baddour J, Achreja A, Bernard V, Moss T, et al. Tumor microenvironment derived exosomes pleiotropically modulate cancer cell metabolism. Elife (2016) 5:e10250. doi:10.7554/eLife.10250

14. Brauer HA, Makowski L, Hoadley KA, Casbas-Hernandez P, Lang LJ, RomanPerez E, et al. Impact of tumor microenvironment and epithelial phenotypes on metabolism in breast cancer. Clin Cancer Res (2013) 19(3):571-85. doi:10.1158/1078-0432.CCR-12-2123

15. Yang L, Achreja A, Yeung TL, Mangala LS, Jiang D, Han C, et al. Targeting stromal glutamine synthetase in tumors disrupts tumor microenvironmentregulated cancer cell growth. Cell Metab (2016) 24(5):685-700. doi:10.1016/ j.cmet.2016.10.011

16. Achreja A, Zhao H, Yang L, Yun TH, Marini J, Nagrath D. Exo-MFA a 13C metabolic flux analysis framework to dissect tumor microenvironmentsecreted exosome contributions towards cancer cell metabolism. Metab Eng (2017) 43(Pt B):156-72. doi:10.1016/j.ymben.2017.01.001

17. Guillaumond F, Leca J, Olivares O, Lavaut MN, Vidal N, Berthezene P, et al. Strengthened glycolysis under hypoxia supports tumor symbiosis and hexosamine biosynthesis in pancreatic adenocarcinoma. Proc Natl Acad Sci U S A (2013) 110(10):3919-24. doi:10.1073/pnas.1219555110

18. Faubert B, Li KY, Cai L, Hensley CT, Kim J, Zacharias LG, et al. Lactate metabolism in human lung tumors. Cell (2017) 171(2):358-71.e359. doi:10.1016/ j.cell.2017.09.019

19. Allen E, Mieville P, Warren CM, Saghafinia S, Li L, Peng MW, et al. Metabolic symbiosis enables adaptive resistance to anti-angiogenic therapy that is dependent on mTOR signaling. Cell Rep (2016) 15(6):1144-60. doi:10.1016/ j.celrep.2016.04.029

20. Lyssiotis CA, Kimmelman AC. Metabolic interactions in the tumor microenvironment. Trends Cell Biol (2017) 27(11):863-75. doi:10.1016/j.tcb.2017. 06.003

21. Feillet C, van der Horst GT, Levi F, Rand DA, Delaunay F. Coupling between the circadian clock and cell cycle oscillators: implication for healthy cells and malignant growth. Front Neurol (2015) 6:96. doi:10.3389/fneur.2015. 00096

22. Krishnaiah SY, Wu G, Altman BJ, Growe J, Rhoades SD, Coldren F, et al. Clock regulation of metabolites reveals coupling between transcription and metabolism. Cell Metab (2017) 25(4):961-74.e964. doi:10.1016/j.cmet.2017. 03.019

23. Ballesta A, Innominato PF, Dallmann R, Rand DA, Levi FA. Systems chronotherapeutics. Pharmacol Rev (2017) 69(2):161-99. doi:10.1124/pr.116. 013441

24. Commisso C, Davidson SM, Soydaner-Azeloglu RG, Parker SJ, Kamphorst JJ, Hackett S, et al. Macropinocytosis of protein is an amino acid supply route in Ras-transformed cells. Nature (2013) 497(7451):633-7. doi:10.1038/ nature 12138
25. Davidson SM, Jonas O, Keibler MA, Hou HW, Luengo A, Mayers JR, et al. Direct evidence for cancer-cell-autonomous extracellular protein catabolism in pancreatic tumors. Nat Med (2017) 23(2):235-41. doi:10.1038/nm.4256

26. Kamphorst JJ, Nofal M, Commisso C, Hackett SR, Lu W, Grabocka E, et al. Human pancreatic cancer tumors are nutrient poor and tumor cells actively scavenge extracellular protein. Cancer Res (2015) 75(3):544-53. doi:10.1158/0008-5472.CAN-14-2211

27. Olivares O, Mayers JR, Gouirand V, Torrence ME, Gicquel T, Borge L, et al. Collagen-derived proline promotes pancreatic ductal adenocarcinoma cell survival under nutrient limited conditions. Nat Commun (2017) 8:16031. doi:10.1038/ncomms16031

28. Schug ZT, Peck B, Jones DT, Zhang Q, Grosskurth S, Alam IS, et al. Acetyl-CoA synthetase 2 promotes acetate utilization and maintains cancer cell growth under metabolic stress. Cancer Cell (2015) 27(1):57-71. doi:10.1016/ j.ccell.2014.12.002

29. Huang D, Li T, Wang L, Zhang L, Yan R, Li K, et al. Hepatocellular carcinoma redirects to ketolysis for progression under nutrition deprivation stress. Cell Res (2016) 26(10):1112-30. doi:10.1038/cr.2016.109

30. Kang HB, Fan J, Lin R, Elf S, Ji Q, Zhao L, et al. Metabolic rewiring by oncogenic BRAF V600E links ketogenesis pathway to BRAF-MEK1 signaling. Mol Cell (2015) 59(3):345-58. doi:10.1016/j.molcel.2015.05.037

31. Xia S, Lin R, Jin L, Zhao L, Kang HB, Pan Y, et al. Prevention of dietary-fatfueled ketogenesis attenuates BRAF V600E tumor growth. Cell Metab (2017) 25(2):358-73. doi:10.1016/j.cmet.2016.12.010

32. Huang CK, Chang PH, Kuo WH, Chen CL, Jeng YM, Chang KJ, et al. Adipocytes promote malignant growth of breast tumours with monocarboxylate transporter 2 expression via beta-hydroxybutyrate. Nat Commun (2017) 8:14706. doi:10.1038/ncomms14706

33. Spinelli JB, Yoon H, Ringel AE, Jeanfavre S, Clish CB, Haigis MC. Metabolic recycling of ammonia via glutamate dehydrogenase supports breast cancer biomass. Science (2017) 358(6365):941-6. doi:10.1126/science.aam9305

34. Egeblad M, Nakasone ES, Werb Z. Tumors as organs: complex tissues that interface with the entire organism. Dev Cell (2010) 18(6):884-901. doi:10.1016/j.devcel.2010.05.012

35. Whatcott CJ, Diep CH, Jiang P, Watanabe A, LoBello J, Sima C, et al. Desmoplasia in primary tumors and metastatic lesions of pancreatic cancer. Clin Cancer Res (2015) 21(15):3561-8. doi:10.1158/1078-0432.CCR-14-1051

36. Christen S, Lorendeau D, Schmieder R, Broekaert D, Metzger K, Veys K, et al. Breast cancer-derived lung metastases show increased pyruvate carboxylase-dependent anaplerosis. Cell Rep (2016) 17(3):837-48. doi:10.1016/j. celrep.2016.09.042

37. Loo JM, Scherl A, Nguyen A, Man FY, Weinberg E, Zeng Z, et al. Extracellular metabolic energetics can promote cancer progression. Cell (2015) 160(3): 393-406. doi:10.1016/j.cell.2014.12.018

38. McDonald OG, Li X, Saunders T, Tryggvadottir R, Mentch SJ, Warmoes MO, et al. Epigenomic reprogramming during pancreatic cancer progression links anabolic glucose metabolism to distant metastasis. Nat Genet (2017) 49(3):367-76. doi:10.1038/ng.3753

39. Fong MY, Zhou W, Liu L, Alontaga AY, Chandra M, Ashby J, et al. Breastcancer-secreted miR-122 reprograms glucose metabolism in premetastatic niche to promote metastasis. Nat Cell Biol (2015) 17(2):183-94. doi:10.1038/ ncb3094

Conflict of Interest Statement: The authors declare that the research was conducted in the absence of any commercial or financial relationships that could be construed as a potential conflict of interest.

Copyright (9) 2018 Gouirand, Guillaumond and Vasseur. This is an open-access article distributed under the terms of the Creative Commons Attribution License (CC BY). The use, distribution or reproduction in other forums is permitted, provided the original author(s) and the copyright owner are credited and that the original publication in this journal is cited, in accordance with accepted academic practice. No use, distribution or reproduction is permitted which does not comply with these terms. 